\title{
THE DIFFERENCE BETWEEN THE INTEGRAL CHARACTERISTICS OF TWO AND THREE WATER DROPLETS MOVING SEQUENTIALLY THROUGH HIGH- TEMPERATURE COMBUSTION PRODUCTS AND AIR
}

\author{
Roman S. Volkov ${ }^{1}$, Ivan S. Voitkov ${ }^{1}$, Maxim V. Zabelin ${ }^{1}$, Pavel A. Kuibin ${ }^{2}$, Pavel A. Strizhak ${ }^{1 \mathrm{a}}$ \\ ${ }^{1}$ National Research Tomsk Polytechnic University, 634050 Tomsk, Russia \\ ${ }^{2}$ Kutateladze Institute of Thermophysics, 630090 Novosibirsk, Russia
}

\begin{abstract}
This paper focuses on the study of macroscopic regularities that describe how moving droplets influence on the evaporation of subsequent droplets in high-temperature gases $(1100 \mathrm{~K}$ ) and air at moderate temperatures (about $300 \mathrm{~K}$ ). Experiments have been conducted by means of high-speed video recording and using specialized software. Investigations have been performed for two and three successive droplets with the initial radius of $1.5 \mathrm{~mm}$. The effect of droplet braking, coagulation and acceleration has been defined. The typical difference between velocities, sizes and distances of successive droplets has been demonstrated.
\end{abstract}

\section{Introduction}

The systems of thermal (as a rule, high-temperature) treatment of water (or emulsions and suspensions based on it) are implemented using different gas flows [1-3]. The most common sources of gas flow are the combustion products of liquid and gaseous fuels, or heated air. The main disadvantage of the modern systems of thermal water treatment is high fuel (or air) consumption needed for liquid droplet evaporation. Another disadvantage is the need for re-transmission droplets through heating chambers. This is due to the significant entrainment of water by gases, its precipitation and condensation on the walls of heating chambers. The entrainment of water by combustion products is also an important problem for the modern systems of fire extinguishing by sprayed flow [4-6].

Droplet sizes in systems, reviewed in studies [1-6], vary from 50 microns to 500 microns. Works [7-9] perform the results of experimental studies, illustrating the necessary and sufficient conditions for the entrainment of water droplets by high-temperature gases. Studies [7-9] demonstrated that droplets with a size less than $200 \mu \mathrm{m}$ are almost completely carried away by typical combustion products. This occurs under conditions corresponding to the systems of thermal water treatment [1-3] and polydisperse extinguishing [4-6]. The analysis of studies [4-6] allowed us to formulate hypotheses about the causes of the entrainment of droplets and their braking by high-temperature combustion products. In particular, works [4-6] emphasized that the conditions of evaporation and braking for the first droplet differ significantly from that for successive droplets. However, experimental confirmations or refutations of that have not yet been published. It is of interest to

${ }^{a}$ Corresponding author: pavelspa@tpu.ru 
perform the experimental investigations of regularities for several water droplets moving sequentially through high-temperature gases, typical for systems as in studies [1-6].

The aim of this work is the experimental study of the main differences between the integral characteristics for the case of two and three water droplets moving sequentially through hightemperature combustion products and air.

\section{Experimental Setup and Procedure}

A setup described on Fig. 1 was similar to facilities that we used in our previous studies [7-9], but it was equipped with a number of specific tools for experiments. The main elements of the setup are: motorized pointing devices 8 with controlled rate and the ability to install remote control cameras, a device for the sequential supply of water droplets 11 with an adjustable distance between droplets released from it, high-speed video cameras 1 "Phantom V411" and "Phantom Miro M310" with a maximum frame frequency up to $6 \cdot 10^{5} \mathrm{fps}$, a laboratory microbalance 6 "ViBRA HT 84RCE" with a resolution $10^{-5} \mathrm{~g}$, an electronic single-channel pipette "Finnpipette Novus" 5 (functional range from 10 $\mu \mathrm{L}$ to $100 \mu \mathrm{L}$, increments $-0.1 \mu \mathrm{L}$ ), a workstation 3 for data processing and the analysis of results.

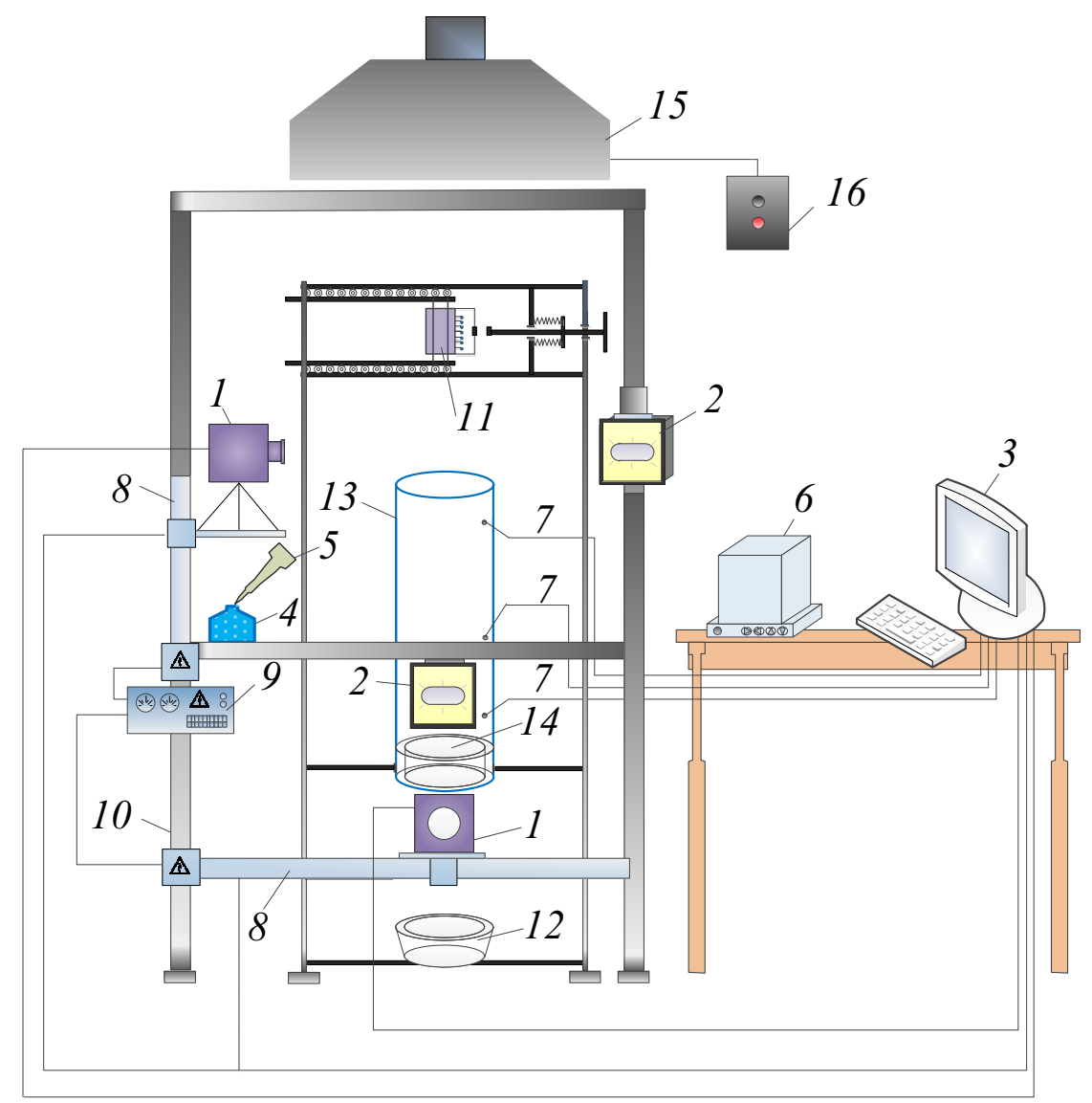

Figure 1. Scheme of an experimental setup : 1 - high-speed video cameras; 2 - spotlights; 3 - personal computer; 4 - container with water; 5 - pipette; 6 - microbalance; 7 - thermocouples; 8 - motorized pointing devices; 9 power supply for multichannel technological registrar; 10 - aluminum rack; 11 - device for sequential droplet supply; 12 - water catcher; 13 - quartz tube; 14 - hollow cylinder with flammable liquid; 15 - air flow system; 16 - remote on/off switch 
Each experiment included a sequence of the following procedures:

- drawing off water $(100 \mu \mathrm{L})$ from a water tank 4 using the pipette 5;

- $\quad$ setting the functional volume of droplets in the pipette 5;

- check weighing of one droplet using a microbalance 6 , and then calculating its volume (to determine the accuracy of pipetting);

- pouring droplets to the needles of a device for sequential droplet supply 11 using the pipette 5 (needles were placed vertically at a fixed distance one above the other);

- bringing the device for sequential droplet supply 11 to the working position by mechanical manipulations;

- releasing droplets into the cylinder 13 ;

- video recording of moving water droplets by high-speed video cameras 1 (the observed area was illuminated by a spotlight 2 for the best display of droplets on videograms);

- video processing and data analysis in workstation 3 with the help of specialized software.

The distance between released water droplets was set in the device 11. The distance varied from 8 to $36 \mathrm{~mm}$ with an increment of $4 \mathrm{~mm}$. We conducted two series of experiments. In the first cycle, we investigated the integral characteristics of two water droplets in the sequential motion. In the second cycle, we performed similar studies for three successive droplets. Each series of experiments included two stages: on the first stage, we recorded droplets motion in air, on the second stage - in hightemperature combustion products. The motion of droplets was recorded along the entire length of the cylindrical channel 13. For this purpose, the entire path of droplets motion (the length of the cylindrical channel 13) was divided conditionally into several sections. One of the high-speed video cameras 1 was fixed to a vertically located pointing device 8 . This camera moved downward and recorded a moving water droplet.

As was mentioned above, a water droplet moved through air and high-temperature combustion products. Air temperature $T_{\mathrm{g}}$ was about $300 \mathrm{~K}$. High-temperature gas medium was obtained through the combustion of typical liquid fuel - kerosene (TS-1). A hollow cylinder 14 (set at the base of the quartz tube 13) was filled with kerosene in the amount of about $200 \mathrm{~mL}$. After that, piezo ignition of kerosene was initiated. After about 200 seconds, the average temperature in the channel 13 reached about $1100 \mathrm{~K}$.

Gas temperature $T_{\mathrm{g}}$ was measured by chromel-alumel thermocouples 7 (temperature range - 273$1373 \mathrm{~K}$, accuracy $\pm 3 \mathrm{~K}$ ) in several heights of the cylinder 13 (Fig. 1) and was about $1100 \mathrm{~K}$. The maximum random measurement error $T_{\mathrm{g}}$ was $30 \mathrm{~K}$. Combustion rate $U_{\mathrm{g}}$ in the cylinder 13 was controlled by an air flow system 15 . Combustion rate was monitored by a vane anemometer "UnionTest AN110" (the maximum error - $0.1 \mathrm{~m} / \mathrm{s}$ ) and was about $1.5 \mathrm{~m} / \mathrm{s}$. The initial water temperature $T_{\mathrm{w}}$ in the tank 4 was monitored by a platinum thermometer (temperature range $-223-473$ $\mathrm{K}$, systematic error $\pm 0.3 \mathrm{~K}$ ). The maximum random measurement error $T_{\mathrm{w}}$ was $1.5 \mathrm{~K}$.

Data processing and the subsequent analysis of obtained results were performed using special software "Phantom Camera Control" ("PCC"), and a software system with the function of continuous monitoring "Tema Automotive" [10, 11].

Software "PCC" allowed us to calibrate the measuring system on the stage of shooting water droplets, choose the optimum frame frequency and resolution, calculate a scale factor, and to exclude from consideration some parameters, which did not meet the conditions of the experiment. Further application of software complex "Tema Automotive" with special built-in algorithms for continuous tracking "Correlation" and "Circular Symmetry" [10,11] allowed us to visualize the motion path of each droplet in the observed area, to determine the vertical distance between droplets, the horizontal trajectory deflections of droplets, and the speed and size of droplets.

The following parameters were chosen as objective functions for data processing: the speed $u_{\mathrm{d}}$ and size (radius) $r_{\mathrm{d}}$ of droplets, the distance between successive droplets $l_{\mathrm{d}}$. Systematic errors of $l_{\mathrm{d}}$ did not exceed $10^{-5} \mathrm{~m}, u_{\mathrm{d}}-0.02 \mathrm{~m} / \mathrm{s}$. The maximum error of $r_{\mathrm{d}}$ was $0.004 \mathrm{~mm}$. 


\section{Results and discussion}

Fig. 2 shows typical frames with results obtained by video processing for the case of three water droplets in the sequential motion. Captured video processing algorithm was as follows. Software identified moving droplets (Fig. 2a), and then we set the parameters for these droplets. After that, selected in the first stage of processing parameters were displayed in graphs and tables (Fig. 2b) with the help of algorithms "Correlation" and "Circular Symmetry".
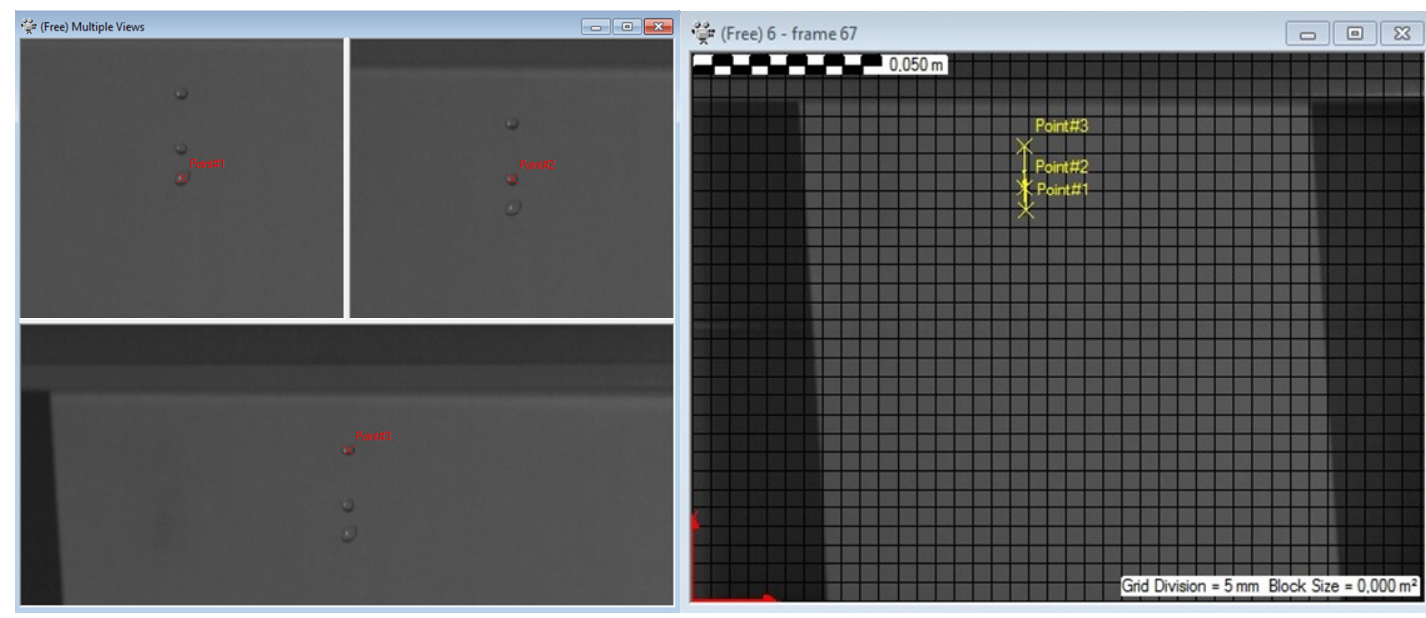

$a$

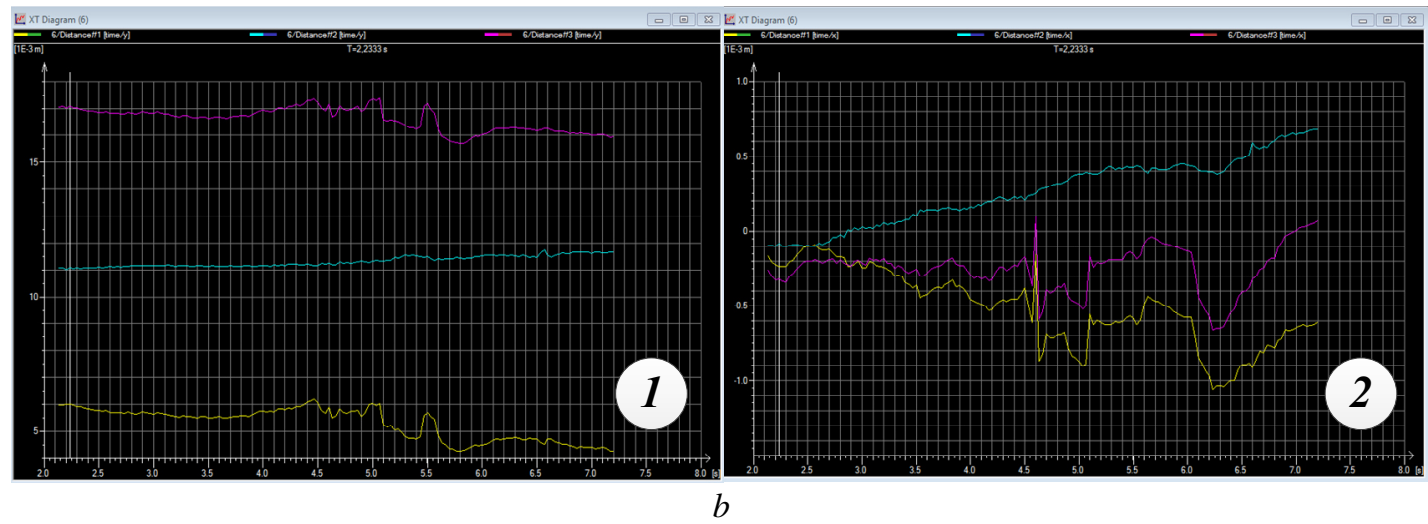

Figure 2. Frames of results obtained by video processing using software "Tema Automotive" for the case of three water droplets in the sequential motion: $a$ - identification of droplets; $b$ - charting the distance between successive droplets in the vertical direction (1), and the trajektory deviation of water droplets motion in the horizontal direction (2)

The same procedure (Fig. 2) was performed for all obtained video recordings in each observed area (over the entire height of the channel 13). The result of such processing was a chart with stretch diagrams. The chart shows a change in the integral characteristics $\left(u_{\mathrm{d}}, l_{\mathrm{d}}, r_{\mathrm{d}}\right)$ of sequentially moving droplets across the length of the channel 13 .

As a result of the analysis of parameters distribution, we plotted the dependence of characteristic distance $l_{\mathrm{d}}$ between two sequentially moving water droplets from the path $L$, which droplets passed through high-temperature combustion products (Fig. 3). 


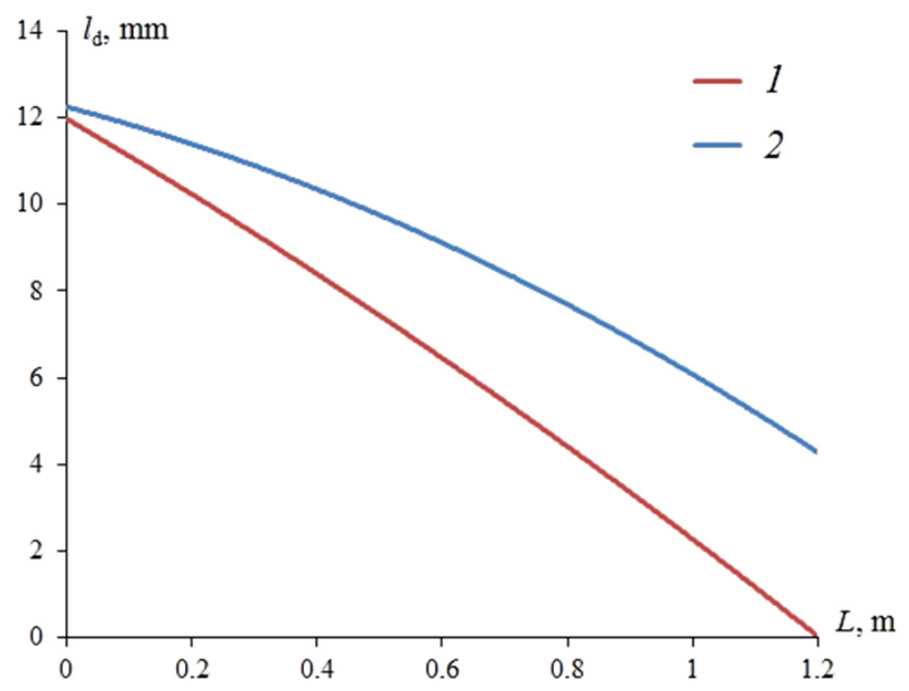

Figure 3. Distance change between two droplets $\left(V_{\mathrm{d} 0}=15 \mu \mathrm{L}, l_{\mathrm{d} 0}=12 \mathrm{~mm}\right)$ in the sequential motion in high-temperature combustion products (1) and air (2)

Results showed (Fig. 3) that the distance between two sequentially moving water droplets, as they moved through high-temperature combustion products, reduced to zero at the distance of $L \approx 1.2 \mathrm{~m}$. Consequently, two liquid droplets coagulate to a one droplet, which continues to move further.

This result can be explained as follows. A water droplet, which is the first ("guiding") in the order, intensively evaporates due to the heating of its subsurface layer to the temperatures of phase transformations. As a result, the "guiding" droplet leaves a vapor trace (steam). In turn, the steam, displaces high-temperature combustion products around the following droplet. Thereby, the "guiding" droplet causes the reduced temperature of the following droplet surface and its slower evaporation. As a result of these processes, the "guiding" droplet brakes, being reduced in weight. The following droplet evaporates much more slowly than the "guiding" droplet. Therefore, the following droplet is less susceptible to braking. Due to this, the distance between these two droplets decreases gradually to zero. It is worth noting that this result correlates well with our previous study [8], where similar processes are demonstrated for fine water droplets $\left(r_{\mathrm{d}}<400 \mathrm{~m}\right)$.

Analyzing the results of the experiments, we discovered an interesting feature. It was found that the processes, occurring during the sequential motion of three or more water droplets, can be accurately described by previously established laws for sequentially moving droplets. Thus, the system of three successive droplets can be described by formula "2"+" 1 ", where " 2 " is the first two moving droplets (under previously established laws), and "1" is a water droplet moving in the steam of the first two droplets (almost no effect on the integral characteristics of first two droplets motion and evaporation). Formula "2"+"1"+"1" can be applied for the system of four sequentially moving droplets, where " 2 " is the first two moving droplets, and " 1 " +1 " is the third and forth liquid droplets moving in the stream of first two droplets. Experiments showed that the previously described processes of droplets convergence and coagulation might be observed for the third and forth droplets, when they moved at the distance of more than approximately $30 \mathrm{~mm}$ from the first two merged droplets.

The table below shows changes in velocity of two and three sequentially moving droplets. The following data were obtained by video processing in software "Tema Automotive".

The analysis of the table shows that, as previously noted, the regularities of convergence are almost identical for two successive water droplets and for the first two droplets from the group of three droplets. It can be seen from the Table that the range of rate changing for the first two droplets (regardless of the presence or absence of the third drop in their tracks). 
Table. Velocities of two and three sequantially moving droplets $\left(V_{\mathrm{d} 0}=20 \mu \mathrm{L}, l_{\mathrm{d} 0}=12 \mathrm{~mm}\right)$ in high-temperature $\left(T_{\mathrm{g}}=1100 \mathrm{~K}\right)$ combustion products (the velocity of coagulated droplets is in italics)

\begin{tabular}{|c|c|c|c|c|c|}
\hline \multirow{2}{*}{$L, \mathrm{~m}$} & \multicolumn{2}{|c|}{ Two droplets } & \multicolumn{3}{c|}{ Three droplets } \\
\cline { 2 - 6 } & 1 & 2 & 1 & 2 & 3 \\
\hline 0 & 0.5 & 0.5 & 0.5 & 0.5 & 0.5 \\
\hline 0.2 & 2 & 2.01 & 2.01 & 2.02 & 2.02 \\
\hline 0.4 & 3.11 & 3.18 & 3.11 & 3.18 & 3.19 \\
\hline 0.6 & 3.75 & 3.86 & 3.75 & 3.85 & 3.86 \\
\hline 0.8 & 4.06 & 4.15 & 4.07 & 4.16 & 4.17 \\
\hline 1 & 4.21 & 4.26 & 4.21 & 4.25 & 4.27 \\
\hline 1.2 & \multicolumn{3}{|c|}{4.31} & \multicolumn{3}{c|}{4.33} & 4.32 \\
\hline 1.4 & 4.55 & 4.56 & 4.45 \\
\hline
\end{tabular}

\section{Conclusion}

The experiments showed that the conditions of the heating and evaporation of sequential droplets differ compared to the first droplet. The scale of differences is determined by the initial distance between droplets. The revealed features of the braking of sequentially moving droplets prove the hypothesis of studies [7-9] and supplement the results of these studies. The developed technique and established effects can be used for solving a large group of fundamental gas-vapor-droplet problems, as well as for prognostic assessment of evaporation completeness and droplets entrainment extent in thermal water treatment and polydisperse fire extinguishing systems.

The study was financially supported by Russian Foundation for Basis Research grant (project 15-38-50122).

\section{References}

1. D. I. Kofman, M. M. Vostrikov, A. V. Antonenko, Chem. Petrol. Eng+ 45, 577 (2009).

2. A.Yu. Valdberg, K.P. Makeeva, Chem. Petrol. Eng+ 46, 305 (2010).

3. A. Yu. Varaksin, High Temp. 51, 377 (2013).

4. X.K. Xiao, B.H. Cong, X.S. Wang, K.Q. Kuang, Richard K.K. Yuen, G.X. Liao, J. Fire Sci. 29, 339 (2011).

5. B. Yao, B.H. Cong, Fire Safety J. 47, 32 (2012).

6. Z. Tang, Z. Fang, J.P. Yuan, B. Merci, Fire Safety J. 55, 35 (2013).

7. R.S. Volkov, G.V. Kuznetsov, P.A. Strizhak, Int. J. Heat Mass Tran. 79, 838 (2014).

8. R.S. Volkov, G.V. Kuznetsov, P.A. Strizhak, Thermophys Aeromech+ 21, 255 (2014).

9. R.S. Volkov, G.V. Kuznetsov, P.A. Strizhak, Int. J. Heat Mass Tran. 88, 193 (2015).

10. J. Janiszewski, Metrol. and Measur. Sys. 19, 797 (2012).

11. J. Janiszewski, Int. J. Solids and Struct. 49, 1001 (2012). 